\title{
MANEJO DA INFECÇÃO APÓS CIRURGIA DE FIXAÇÃO INTERNA DA COLUNA LOMBAR
}

\author{
Asdrubal Falavigna', Orlando Righesso Neto², \\ Gabriela Poglia Fonseca ${ }^{3}$, Monique Nervo
}

\begin{abstract}
RESUMO - A incidência de infecções profundas em cirurgias de coluna lombar situa-se na faixa de 0,7\% e $11,6 \%$, sendo uma das causas de morbidade na fase aguda deste procedimento. 0 objetivo deste estudo foi avaliar o tratamento da infecção após cirurgia de fixação interna de coluna lombar. Foram analisados 260 pacientes que se submeteram à cirurgia de fixação posterior da coluna lombar com instrumentação e enxertia de osso ilíaco no período de janeiro de 1997 a janeiro de 2005. A infecção pós-operatória ocorreu em oito ( $3 \%$ ) casos. A idade média dos pacientes foi 56 anos, com maior prevalência do sexo masculino ( 5 casos). Staphylococcus aureus foi isolado em $85 \%$ dos pacientes (6 casos). 0 tratamento instituído foi a lavagem externa e a antibioticoterapia intravenosa, seguida pela via oral. O tempo médio de hospitalização dos pacientes foi 35,8 dias. Com o tratamento preconizado foi possível erradicar a infecção sem a necessidade da retirada do sistema de fixação interna em todos os casos.
\end{abstract}

PALAVRAS-CHAVE: infecção, instrumentação, antibioticoterapia.

\begin{abstract}
Management of deep wound infections in spinal lumbar fusions
ABSTRACT - The rate of deep wound infections in spinal lumbar fusions is around $0.7 \%$ to $11.6 \%$, being one of the causes of morbidity in acute phase. The aim of this study was to evaluate the management of spinal infection after internal lumbar fusions. Two hundred and sixty patients, who underwent to spinal surg ery with lumbar fusion and iliac bone grafting, were analized, from January 1997 to January 2005. Wound infection was observed in eight (3\%) cases. The average of age was 56 years, with a higher prevalence in males ( 5 patients). Most prevalent was Staphylococcus aureus in 6 patients. The treatment was done by intravenous antibiotic therapy folowed by oral therapy and local irrigation. The average time of hospitalization was 35.8 days. It was possible to erradicate infection without removal of instrumentation in all patients.
\end{abstract}

KEY WORDS: infection, instrumentation, antibiotic therapy.

A infecção é uma das causas de morbidade na fase aguda após ciru rgias da coluna lombar, resultando em hospitalização prolongada e aumento dos custos, podendo comprometer o resultado da cirurgia se não for tratada adequadamente ${ }^{1,2}$. A prevenção através de técnicas de assepsia e de antimicrobianos profiláticos continua sendo a alternativa mais adequada ${ }^{1,2}$. A incidência de infecções profundas em ciru rgias da coluna lombar situa-se entre $0,7 \%$ a $11,6 \%{ }^{3-7}$.

$\mathrm{O}$ uso de material de fixação interna vem acompanhado de maior taxa de infecção, não havendo consenso sobre o tratamento de escolha: antibioticoterapia, lavagem externa, retirada da instrumentação, remoção do enxerto ósseo, fechamento por segunda intenção ou abertura diária para limpeza ${ }^{6-18}$.
Este estudo tem o objetivo de avaliar a incidência e o manejo da infecção após fixação interna da coluna lombar.

\section{MÉTODO}

Foram estudados ret rospectivamente os pacientes que se submeteram à ciru rgia de fixação posterior da coluna lombar com instrumentação e enxertia de osso ilíaco no período de janeiro de 1997 a janeiro de 2005 e que apresentaram infecção cirúrgica. O material de fixação utilizado foi parafuso pedicular associado ou não com espaçador intersomático. A antibiotico profilaxia foi introduzida em todos os casos usando-se cefazoli na, e a equipe cirúrgica foi a mesma em todos procedimentos. O diagnóstico foi definido pelo crescimento bacteriano na secreção da ferida operatória.

'Professor da Disciplina de Neurologia, Departamento de Neurologia da Universidade de Caxias do Sul, Caxias do Sul RS, Brasil (UCS); ${ }^{2}$ O rtopedista, Pós-Graduando em Ortopedia, Faculdade de Ciências Médicas da Santa Casa de São Paulo, São Paulo SP, Brasil (FMSCSP); ${ }^{3}$ Aluna do $11^{\circ}$ semestre do Curso de Medicina da UCS.

Recebido 10 Maio 2006, recebido na forma final 11 Julho 2006. Aceito 16 Agosto 2006.

Dr. Asdrubal Falavigna - Rua General Arcy da Rocha Nóbrega 401 / 602 - 95040-290 Caxias do Sul RS - Brasil. E-mail: asdruba/@doctor.com 
Con firmeda a infecção, os pacientes foram submetidos a revisão da ciru rgia, lavagem exaustiva com coleta de material para exame bacteriológico, e remoção dos fragmentos ósseos desvitalizados e do tecido necrótico. O material de fixação foi mantido, sendo instalado um sistema de lavagem contínua com um dreno porto-vac conectado ao equipo de solução fisiológica a $0,9 \%$ com gotejo de 40 gotas por minuto e um dreno de tórax conectado ao sistema de frascos coletores, ligados à rede de vácuo para retirada do líquido. O sistema de lavagem foi mantido por 5 dias, podendo ser recolocado conforme a necessidade. Simultaneamente, foi instituída antibioticoterapia intravenosa e, a seguir por via oral, de acordo com a sensibilidade do microorganismo.

A resposta ao tratamento e a duração da antibioticoterapia foram monitoradas através de leucograma, velocidade de sedimentação globular e proteína $C$ reativa.

\section{RESULTADOS}

A infecção pós-operatória foi observada em oito (3\%) dos 260 casos operados para fixação posterior lombar com instrumentação e enxertia óssea de ilíaco nos últimos oito anos. A idade média dos pacientes acometidos foi 56 anos, com maior prevalência no sexo masculino ( 5 masculinos e 3 femininos). As informações sobre esses oito pacientes estão contidas na Tabela.

O tempo médio de aparecimento dos sintomas foi 16 dias, havendo apenas um caso com sintomatologia mais tardia (36 semanas). Todos apresentaram secreção pela ferida operatória com crescimento de microorganismos no exame cultural. Foi necessário recolocar o sistema de lavagem em cinco casos, um deles necessitando deste tratamento por três vezes. Nos outros casos, a infecção foi erradicada somente com um ciclo de lavagem local.

O agente etiológico mais prevalente foi oStaphy lococcus aureus em seis pacientes. Antibioticoterapia intravenosa foi mantida por 4 a 6 semanas e complementada, com administração por via oral por, no máximo, 2 semanas.

O tempo médio de hospitalização dos pacientes foi 35,8 dias ( 28 a 42 dias).

\section{DISCUSSÃO}

Múltiplos fatores têm sido associados ao surg imento de processo infeccioso nas cirurgias da coluna, tais como idade avançada, obesidade, tabagismo, diabetes, repouso prolongado, imunossupressão, má nutrição e processos infecciosos a distância ${ }^{9,19}$. Desnutrição e tabagismo são causas importantes de predisposição à infecção. A baixa ingesta protéica está associada à imunossupressão e à dificuldade de cicatrização, sendo ideal que o nível de albumina seja superior a 3,5 g/dL e que a contagem de linfócitos se situe ent re 1500-2000 células $/ \mathrm{mm}^{3}$ para reduzirem-se os risco de infecçãa $0^{1,20}$. Os pacientes fumantes têm incidência nove vezes maior de apresentar infecção quando comparados aos não fumantes ${ }^{8}$. Isoladamente, a idade não parece ser um fator preditor de infecção ${ }^{18,21}$.

Fatores relacionados com a ciru rgia também pro-

Tabela. Apresentação dos casos de infecção após cirurgia da coluna lombar com uso de instrumental.

\begin{tabular}{|c|c|c|c|c|c|c|c|c|c|c|}
\hline Caso & Sexo & $\begin{array}{l}\text { Idade } \\
\text { (anos) }\end{array}$ & $\begin{array}{l}\text { Doença } \\
\text { de base }\end{array}$ & Tipo germe & $\begin{array}{c}\text { Fatores } \\
\text { predisponentes }\end{array}$ & $\begin{array}{c}\text { Tempo } \\
\text { diagnóstico } \\
\text { (semanas) }\end{array}$ & $\begin{array}{c}\text { Tempo } \\
\text { hospitalização } \\
\text { (semanas) }\end{array}$ & $\begin{array}{c}\text { Lavagens } \\
\text { externas } \\
\text { (dias) }\end{array}$ & $\begin{array}{c}\text { ATB IV } \\
\text { (semanas) }\end{array}$ & $\begin{array}{c}\text { ATB VO } \\
\text { (semanas) }\end{array}$ \\
\hline 1 & $\mathrm{~F}$ & 53 & $\begin{array}{l}\text { Discopatia e } \\
\text { Hérnia discal }\end{array}$ & $\begin{array}{c}\text { Staphyloccocus } \\
\text { aureus }\end{array}$ & $\begin{array}{c}\text { Diabetes mellitus } \\
\text { Tabagismo }\end{array}$ & 2 & 4 & 10 & 4 & 1 \\
\hline 2 & $\mathrm{~F}$ & 74 & $\begin{array}{l}\text { Estenose } \\
\text { do canal }\end{array}$ & $\begin{array}{c}\text { Staphyloccocus } \\
\text { aureus }\end{array}$ & $\begin{array}{l}\text { Obesidade } \\
\text { Tabagismo }\end{array}$ & 2 & 5 & 10 & 5 & 1 \\
\hline 3 & M & 55 & $\begin{array}{l}\text { Discopatia e } \\
\text { Hérnia Discal }\end{array}$ & $\begin{array}{c}\text { Staphyloccocus } \\
\text { aureus }\end{array}$ & $\begin{array}{c}\text { Diabetes mellitus } \\
\text { Obesidade }\end{array}$ & 3 & 6 & 10 & 6 & 2 \\
\hline 4 & $M$ & 41 & $\begin{array}{l}\text { Fratura } \\
\text { em L5 }\end{array}$ & $\begin{array}{c}\text { Staphylococcus } \\
\text { species + } \\
\text { Enterobacter }\end{array}$ & - & 1 & 4 & 5 & 4 & 2 \\
\hline 5 & $\mathrm{~F}$ & 69 & $\begin{array}{l}\text { Estenose } \\
\text { do canal }\end{array}$ & $\begin{array}{c}\text { Staphylococcus } \\
\text { species }\end{array}$ & - & 4 & 6 & 15 & 6 & 2 \\
\hline 6 & $M$ & 39 & Discopatia & $\begin{array}{c}\text { Staphyloccocus } \\
\text { aureus }\end{array}$ & $\begin{array}{c}\text { Cirugia prévia } \\
\text { Obesidade }\end{array}$ & 2 & 5 & 5 & 4 & 1 \\
\hline 7 & $M$ & 67 & $\begin{array}{l}\text { Estenose } \\
\text { do canal }\end{array}$ & $\begin{array}{c}\text { Staphyloccocus } \\
\text { aureus }\end{array}$ & $\begin{array}{l}\text { Obesidade } \\
\text { Tabagismo }\end{array}$ & 36 & 5 & 5 & 4 & 2 \\
\hline 8 & $M$ & 53 & Discopatia & $\begin{array}{c}\text { Staphyloccocus } \\
\text { aureus }\end{array}$ & $\begin{array}{c}\text { Diabetes mellitus } \\
\text { Tabagismo }\end{array}$ & 2 & 6 & 10 & 6 & 2 \\
\hline
\end{tabular}

ATB IV, antibioticoterapia intravenosa; ATB VO, antibioticoterapia via oral. 
piciam maior risco de infecção pós-operatória, sendo eles: duração superior a cinco horas, retração prolongada, conversa e tráfego excessivo de pessoas na sala de operação, uso de enxertos ósseos, perda sangüínea acima de $1000 \mathrm{~mL}$, tempo de hospitalização prévio à cirurgia e uso de instrumentação $0^{8,22,23}$.

O tipo de ciru rgia talvez seja o fator mais importante a afetar as taxas de infecção, sendo que as infecções ocorrem predominantemente em procedimentos realizados pela via posterior e com o uso de instrumentação 24,25 .

O sucesso terapêutico começa por um diagnóstico precoce ${ }^{18}$. No pós-operatório, usualmente os pacientes queixam-se de desconforto lombar relacionado com a dissecção muscular. Esse desconforto tende a aliviar nas primeiras duas semanas; entretanto, se a dor aumentar ou retornar após período de acalmia, deve ser cogitada a hipótese de infecção ${ }^{3}$. O sintoma dolo roso geralmente se manifesta nas primeiras três semanas após o procedimento cirúrgico, podendo estar associado a temperatura elevada, edema, eritema ou descamação da ferida pós-operatória ${ }^{4}$. A drenagem de secreção purulenta pela ferida ocorre mais tardiamente, estando presente em $93 \%$ dos pacientes no momento do diagnóstico ${ }^{3,18}$. As infecções são comumente diagnosticadas entre 4 e 45 dias (média 16 dias), após o procedimento cirúrgico, sendo possível detectar-se infecções a distância devido à disseminação hematogênica ${ }^{14}$. As infecções podem ser classificadas em superficiais, em que a pele e o tecido subcutâneo são acometidos, ou profundas, se comprometerema fáscia ou as camadas musculares, sendo difícil determinar sua extensão sem a exploração cirúrgica ${ }^{5,26}$. Mais de $80 \%$ das infecções detectadas são profundas, o que aumenta a morbidade, o tempo de permanência no hospital e a necessidade de reinternação e até mesmo de nova intervenção cirúrgica ${ }^{25}$.

Os exames laboratoriais, como leucograma, velocidade de sedimentação globular e proteína $\mathrm{C}$ reativa, são sensíveis, porém não específicos, podendo ser parâmetros no estabelecimento do diagnóstico de infecção e na eficácia do tratamento ${ }^{18,23}$. Os estudos de imagem, tomografia computadorizada e ressonância magnética, têm limitação pela presença de artefato dos dispositivos metálicos ${ }^{1,5}$. Staphylococcus au reus foi o microorganismo mais encontrado nas infecções pós-operatórias e, em segundo lugar, Staphy lococcus epidermidis 1,2,5,9,26. Outros germes também foram apontados como responsáveis pelas infecções, como o Enterobacter cloacae, Escherichia coli e Ente - rococcus sp, sendo comum a associação de Staphylo coccus aureus e Peptococcus, Staphylococcus aure us e Enterococcus, Staphylococcus epidermidis ePseu domonas ${ }^{4,25}$.

A eficácia da antibioticoprofilaxia em ciru rgias de coluna é bem estabelecida, possibilitando redução da taxa de infecção de $9,3 \%$ para $2,8 \%$. É sugerido o uso de cefalosporinas uma hora antes da cirurgia até 24 horas após o procedimento. $O$ antibiótico profilático mais prescrito foi a cefazolina na dose de 2 $\mathrm{g} / \mathrm{dia}^{1,2,9,26}$. A antibioticoprofilaxia deve ser escolhida de acordo com as características de cada paciente e com os fatores de risco envolvidos, sugerindo-se, em casos especiais, a associação de cefazolina com gentamicina ou com vancomicina ${ }^{4}$. A terapia ideal é determinada pela extensão da infecção e pelo envolvimento do enxerto e do material de síntese ${ }^{5}$. O manejo deve ser feito através de debridamento do tecido necrosado, limpeza, irrigação exaustiva e antibioticoterapia após seis a oito semanas de acordo com os testes de sensibilidade do microorganismo ${ }^{4,5}$. $\mathrm{Na}$ infecção profunda, recomenda-se a utilização de sistema de irrigação contínua por 5 a 10 dias, podendose repetir conforme a necessidade ${ }^{1,2}$. Todos os pacientes devem receber antibioticoterapia por cerca de seis semanas de acordo com os testes de sensibilidade aos antimicrobianos, e um novo debridamento deve ser realizado se houver necessidade, dependendo da extensão e da aparência da lesão e do microorganismo causador ${ }^{1,25,26}$.

Glassman e col. ${ }^{4}$ utilizam antibióticos impregnados com grânulos de polimetilmetacilato fixados em fios de metal. $O$ deslocamento dos grânulos laterais para a instrumentação previne a migração da infecção para a área do canal neural. A fascia profunda é aproximada e nela são colocados os grânulos com antibióticos superficialmente e inserido um dreno. Esta técnica é usada como tratamento adjunto à irrigação e ao debridamento, a fim de obter-se controle das infecções sem a necessidade de remoção do material de fixação. Outros autores adotam a irrigação com solução salina antibacitracina durante o procedimento inicial e descartam a necessidade do uso de siste-

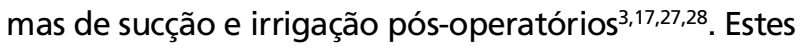
sistemas podem acarretar maior risco de pseudoartose e superinfecção por Pseudomonas, assim como a impossibilitar a realização de debridamento de tecido necrótico quando necessário ${ }^{15,29}$.

A principal questão a ser esclarecida sobre a terapia da infecção pós-operatória é a remoção ou não da instrumentação. $O$ uso da instrumentação de colu- 
na lombar por via posterior elevou o índice de infecção de $2 \%$ para $6 \%{ }^{5,9}$. Weinstein e col. afirmam que a instrumentação e os enxertos ósseos devem ser deixados para elevarem ao máximo a chance de fusão ${ }^{3}$. Esta conduta não é consenso, já que, em $35 \%$ dos casos com infecção de coluna lombar, é necessária a remoção da instrumentação para aumentar a chance de erradicação da infecção devido à proliferação de $\mathrm{fi}$ ib roblastos e reação de corpo estranho $0^{5,10,11}$. Outros a uto res preconizam a remoção não só da instrum entação como também do enxerto ósseo $0^{1,3,6,7,15,16}$. Nas infecções tardias, consideram que a remoção da instrumentação é mais importante em razão da form ação de um "glicocálice", que não pode ser removido sem a retirada da instrumentação, sendo que ele não apenas inibe o sistema imunológico, como também impede a detecção dos microorganismo presentes na instrumentação com as técnicas de aspiração e culturas usadas, sendo o tratamento eficaz nas infecções tardias feito através de irrigação, debridamento, remoção da instrumentação, uso de drenos e administração de antibióticos por 6 semanas ${ }^{12-14}$. Por outro lado, a instrumentação serve para estabilizar o segmento comprometido, teoricamente diminuindo a inflamação e acelerando a cicatrização'. Existem muitos artigos na literatura discordando da conduta de remoção da instrumentação, por prejudicar o resultado da cirurgia $a^{6,7,15,16}$. A instrumentação não deve ser retirada em situações agudas, sendo mantida a fim de atingir a imobilização desejada, uma vez que desalinhamento da coluna, compressão neural e paralisia são complicações devastadoras associadas à perda de estabilidade da coluna, quando o equipamento é removido antes do tempo para adequada fusão ${ }^{17}$. Quando a artrodese estiver consolidada, a instrumentação pode ser removida se necessário ${ }^{2}$. Pseudoartrose pode vir a ocorrer após uma infecção profunda em $30 \%$ a $85 \%$ dos pacientes 6 . Também pode ser apontada como causa de infecção, havendo associação ainda não bem estabelecida entre pseudoatrose e infecção cirúrgica, principalmente quando esta é tardia ${ }^{30}$.

Em conclusão, a infecção após ciru rgia de fixação interna da coluna lombar pose ser tratada segundo o protocolo descrito: debridamento, irrigação local, fechamento primário e antibioticoterapia, sem remoção da instrumentação. Esta opção de tratamento tem como vantagens à de manter a estabilidade do segmento operado, devido a manutenção do material de fixação interna, e evitar o desconforto da limpeza e troca de curativo diário, o que é observado quando se opta por fechamento secundário.

\section{REFERÊNCIAS}

1. Beiner JM, Grauer J, Kwon BK, Vaccaro AR. Postoperative wound infections of the spine. Neurosurg Focus 2003;15:14.

2. Picada R, Winter RB, Lonstein JE, et al. Postoperative deep wound infection in adults after posterior lumbosacral spine fusion with instrumentation: incidence and management. J Spinal Dis 2000;13:42-45.

3. Weinstein MA, McCabe JP, Camissa FP. Postoperative spinal wound infection: a review of 2391 consecutive index procedures. J Spinal Disord 2000;134:422-426.

4. Glassman SD, Dimar JR, Puno RM, et al. Salvage of instrumental lumbar fusions complicated by surgical wound infection. Spine 1996; 21:21632169.

5. Abbey DM, Turner DM, Warson JS, et al. Treatment of postoperative wound infections following spinal fusion with instrumentation. J Spinal Disord 1995;8:278-283.

6. Stambough JL, Beringer D. Postoperative wound infections complicating adult spine surgery. J Spinal Dis 1992;5:277-285.

7. Massie JB, Heller JG, Abitbol JJ, McPherson D, Garfin SR. Postoperative posterior spinal wound infections. Clin Orthop 1992;284:99-108.

8. Thalgott Js, Cotler HB, Sasso RC, et al. Postoperative infections in spinal implants: classification and analysis- A multicenter study. Spine 1991; 16:981-984.

9. Hodges SD, Humphreys SC, Eck JC, Covington LA, Kurzynske NG. Low postoperative infection rates with instrumented lumbar fusion. South Med J 1998;91:1132-1136.

10. Cordero J, Munuera L, Folgueira MP. Influence of metal implants on infection. J Bone Join Surg 1994;5:717-720.

11. Galante JO, Lemous J, Spector M, Wilson J, Wright TM. The biologic effects of implant materials. J Orthop Res 1991;9:760-775.

12. Cristina AG,Costerton JW. Bacterial adherence to biomaterials and tissue. J Bone Joint Surg Am 1985;67:264-273.

13. RichardsBS. Delayed infections following posterior spinal instrumentation for the treatment of idiopathic scoliosis. J Bone Joint Surg Am 1995;77:524-529.

14. Viola RW, King HA, Adler SM, Wilson CB. Delayed infection after elective spinal instrumentation and fusion. Spine 1997;20:2444-2451.

15. Dernbach PD, Gomez H, Hahn J. Primary closure of infected spinal wounds. Neurosurgery 1990;26:707-709.

16. Griffiths HED, Jones DM. Pyogenic infection of the spine: a review of 28 cases. J Bone Joint Surg 1971;53:383-389.

17. Levi ADO, Dickman CA, Sontag VKH. Management of postoperative infections after spinal instrumentation. J Neurosurg 1997;86:975-980.

18. Lonstein J, Winter R, Moe J, et al. Wound infection with Harrington inst rumentation and spine fusion for scoliosis. Clin Orthop 1973;96:222-233.

19. Simpson JM, Silveri CP, Balderston RA, et al.The results of operations on the lumbar spine in patients who have diabetes mellitus. J Bone Joint Surg Am 1993;75:1823-1829.

20. Klein JD,Garfin SR.Nutricional status in the patient with spinal infection. Orthop Clin N Am 1996;27:105-110.

21. Capen DA, Calderone RR, Green A. Perioperative risk factors for wound infections after lower back fusions. Orthop Clin N Am 1996;27: 83-86.

22. Pittet D, Ducel G. Infectious risck factors related to operating rooms. Infect Control Hosp Epidemiol 1994;15:456-462.

23. Thelander U, Larsson S. Quantitition of C-reactive protein lavels and erythrocyte sedimentation rate after spinal surgery. Spine 1992;17: 400-404.

24. Wimmer C, Gluch H, Franzreb M, et al. Predisposing factors for infection in spine surgery: a survey of 850 spinal procedures. J Spinal Disord 1988;11:124-128.

25. Olsen MA,Mayfield J,Lauryssen C, et al. Risk factors for surgical site infection in spinal surgery. J Neurosurg 2003;98:149-155.

26. Wimmer C, Gluch H. Management of postoperative wound infection in posterior spinal fusion with instrumentation. J Spinal Disord 1996; 9:505-508.

27. Garrido E. Closed irrigation-suction technique in the treatment of lumbar laminectomy infection: case report. Neurosurgery 1979;5:354-355.

28. Ido K, Shimizu K, Nakayama Y, et al. Suction/irrigation for deep wound infection after spinal instrumentation: a case study. Eur Spine J 1996; 5:345-349.

29. Transfeldt EE, Lonstein JE, Winter RB, et al. Wound infections in reconstrutive spinal surgery. Orthop Trans Rel Res 1985;9:128.

30. Merrit K, Dowd JD. Role of internal fixation in infection of open fracture: studies with Staphylococcus aureus e Proteus mirabilis. J Orthop Res 1987;5:23-28. 